\title{
Clinical and radiographic features of multiple epiphyseal dysplasia not linked to the COMP or type IX collagen genes
}

\author{
Geert R Mortier*1$^{\star 1}$ Kathryn Chapman ${ }^{2}$, Jules L Leroy ${ }^{1}$ and Michael D Briggs ${ }^{2}$
}

\author{
${ }^{1}$ Department of Medical Genetics, Ghent University Hospital, Ghent, Belgium; ${ }^{2}$ Wellcome Trust Centre for Cell \\ Matrix Research, Manchester, UK
}

Multiple epiphyseal dysplasia (MED) is a mild chondrodysplasia affecting the structural integrity of cartilage and causing early-onset osteoarthrosis in adulthood. The condition is genetically heterogeneous. Mutations in the COMP gene and in two genes (COL9A2; COL9A3), coding respectively for the $\alpha 2$ (IX) and $\alpha 3$ (IX) chains of type IX collagen, can cause the autosomal dominant forms of MED. Mutations in the DTDST gene have recently been identified in a recessive form of MED. However, for the majority of MED cases, the genetic defect still remains undetermined. We report a three-generation family with an autosomal dominant form of MED, characterised by normal stature, joint pain in childhood and early-onset osteoarthrosis, affecting mainly the hips and knees. Based on discordant inheritance among affected individuals linkage of the phenotype to the COMP, COL9A1, COL9A2, COL9A3 genes was excluded. Our study provides evidence that at least another locus, distinct from COL9A1, is involved in autosomal dominant MED. European Journal of Human Genetics (2001) 9, 606-612.

Keywords: multiple epiphyseal dysplasia; osteochondrodysplasia; skeletal dysplasia; genetic linkage

\section{Introduction}

Multiple epiphyseal dysplasia (MED) is a clinically mild and genetically heterogeneous osteochondrodysplasia. The condition usually presents in childhood with complaints of fatigue and pain in the hips and/or knees after exercise. In the initial stage of the disease, radiographs of the skeleton reveal a delay in epiphyseal ossification. Later, small and irregular epiphyses appear, most pronounced in the hips and knees. In adulthood, the disorder is characterised by normal to mild short stature and early-onset osteoarthrosis., ${ }^{1,2}$ The first MED locus identified was the cartilage oligomeric matrix protein (COMP) gene (EDM1, OMIM\#132400). ${ }^{3}$ Mutations in the COMP gene can also cause pseudoachondroplasia, a more severe but related osteochondrodysplasia. ${ }^{4,5}$ Subsequently, mutations in two genes coding

*Correspondence: GR Mortier, Department of Medical Genetics, Ghent University Hospital, De Pintelaan 185, B-9000 Ghent, Belgium.

Tel: +329240 3603; Fax: +329240 4970; E-mail: geert.mortier@rug.ac.be Received 21 February 2001; revised 3 May 2001; accepted 22 May 2001 for the $\alpha$-chains of type IX collagen (COL9A2; COL9A3) were identified in MED patients (EDM2, OMIM\#600204; EDM3, OMIM\#600969). ${ }^{6,7}$ Interestingly, the mutations in the COL9A2 and COL9A3 genes all result in skipping of exon 3 during mRNA processing. EDM1, 2 and 3 all show autosomal dominant inheritance. Recently, homozygosity for a R279W mutation in the diastrophic dysplasia sulphate transporter (DTDST) gene has been shown to cause a recessive form of MED (EDM4, OMIM\#226900). ${ }^{8}$ However, in a significant proportion of MED patients no mutations at the known loci have been identified ${ }^{9,10}$ (KL Chapman and MD Briggs (unpublished observations) and L Ala-Kokko (personal communication)).

In this study we report a family with an autosomal dominant form of MED, in which we excluded linkage to the known loci for autosomal dominant MED (COMP, COL9A2 and COL9A3). Linkage to another candidate gene (the gene encoding the $\alpha 1$ (IX) chain of type IX collagen COL9A1) was also excluded. Our data provide strong evidence for the existence of a fourth locus involved in autosomal dominant MED. 


\section{Materials and methods}

\section{Subjects}

All living relatives in a Belgian, three-generation family with MED were clinically examined. Radiographs of the skeleton were performed in the affected individuals and in those suspected to have the disorder based on clinical grounds. Comprehensive clinical and radiographic evaluation allowed the conclusion that eight family members were indeed affected (Figure 1). After informed consent, all agreed to full cooperation with the study.

\section{Linkage analysis}

Blood samples for DNA analysis were taken and genomic DNA was prepared by standard methods. PCR amplification of microsatellite markers was carried out in $10 \mu \mathrm{l}$ PCR reactions containing $2 \mathrm{mM} \mathrm{MgCl}_{2} ; 10 \mathrm{~mm}$ Tris- $\mathrm{HCl} \mathrm{pH} \mathrm{8.3;} 50 \mathrm{~mm} \mathrm{KCl}$; 0.2 mM each of dATP, dCTP, dGTP and dTTP; 5 pmol of each primer and 50 ng of genomic DNA. Reactions were carried out in a 96-well microtitre plate using a Hybaid PCR express thermal cycler and 35 cycles of $94^{\circ} \mathrm{C}$ for $30 \mathrm{~s}, 57^{\circ} \mathrm{C}$ for $30 \mathrm{~s}$ and $72^{\circ} \mathrm{C}$ for $45 \mathrm{~s}$ were performed. Primer sequences of the newly identified markers were for the COL9A2 marker (atta/gt $)_{n}: 5^{\prime}$ AGGGGAAGGTATTATGCTGGAG-3' (forward) and 5'-GACCAGCCTGGGTCTCAACAC-3' (reverse) and for the COL9A3 marker (CA)n : 5'-GGCATTCCAGAAGGGTTTGCT-3' (forward) and 5'-TTCTGCTGGGCTCCTTAGCTG-3' (reverse).

PCR products were resolved by electrophoresis on a $6 \%$ polyacrylamide gel using an ABI 373A DNA sequencer and data analysed using the ABI 672 genescan collection and genescan PCR analysis software. Genotypes were assigned and LOD scores were calculated using MLINK within LINKAGE version 5.20. For these calculations MED was defined as an autosomal dominant disease with full penetrance.

\section{Results}

Clinical and radiographic findings

We had the opportunity to study the clinical and radiographic phenotype of several affected individuals at different

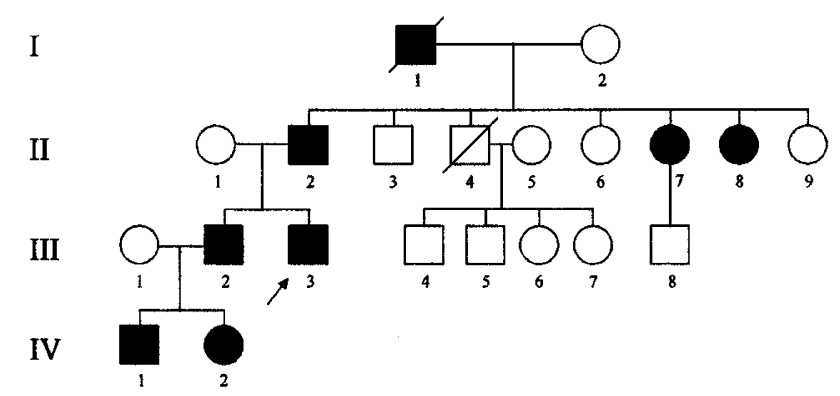

Figure 1 Pedigree of family with MED. Blackened symbols indicate affected individuals. The proband is marked by an arrow. ages (Figure 1). The youngest patient IV-2 was a 19-monthold girl. Her physical examination was normal and she had no clinical complaints. The diagnosis was already suspected at the age of 8 months because of absent ossification of the proximal femoral epiphyses and small and irregular knee epiphyses (Figure 2a). Her older brother IV-1 was also affected with complaints of pain in hips and knees after exercise from the age of 3 years. Physical examination at the age of 5 years revealed a height of $97 \mathrm{~cm}(\mathrm{P} 3=100 \mathrm{~cm})$, normal gait and limited extension at the elbows. Radiographs of the hips and knees demonstrated small and irregularly contoured epiphyses with mild metaphyseal changes (Figures 2b; 3b). Radiographically, the hands were characterised by delayed ossification of normally contoured carpal bones, mild shortening of the metacarpals and phalanges, flattened distal radial epiphysis and irregular distal ulnar metaphysis. The epiphyses of the other long tubular bones were also flattened and delayed in ossification. The spine was normal.

The father III-2 and paternal uncle III-3 (the initial proband of the family) had a congruent history of pain in the knees from early childhood. Both had a normal stature (III-2: $169 \mathrm{~cm}$; III-3: $172 \mathrm{~cm}$ ). Radiographs of III-3 at the age of 12 years showed small proximal femoral epiphyses with fragmentation on the right side (Figure 3c). Radiographs of the knees of III- 2 at 14 years of age revealed flattened epiphyses with striated femoral metaphyses (Figure 2c). The epiphyses of other tubular bones also were affected. Radiographs of the hands of III-3 at 15 years of age were normal. Radiographic evaluation of the grandfather (II-2) revealed dysplasia with secondary osteoarthrosis of hips and knees, but a normal spine. Two paternal grandaunts (II-7, II-8) had similar joint complaints from childhood on. Individual II-7 underwent surgery at the age of 12 years because of a slipped epiphysis at the right hip. On this side hip replacement was performed at the age of 47 years. Individual II- 8 had a knee replacement at the age of 58 years. Radiographs of the hips and knees at the age of 29 years also revealed hip and knee dysplasia (Figure 2d).

\section{Linkage analysis}

In order to determine whether the MED phenotype in this kindred was consistent with linkage to any of the known or candidate MED loci, we used PCR-based genetic linkage analysis with polymorphic markers that were either intragenic, or closely linked, to the tested genes (Table 1). The tested genes were those that encode cartilage oligomeric matrix protein (COMP) and the three constituent chains of type IX collagen (COL9A1, COL9A2 and COL9A3). Because mutations in the type II collagen gene have been reported in families with precocious osteoarthrosis, ${ }^{16}$ linkage to COL2A1 was also performed to establish conclusively that the phenotype did not result from a mutation in the COL2A1 gene.

It became apparent that whilst there were good genetic markers for COMP, COL9A1 and COL9A2, there was not a 
a

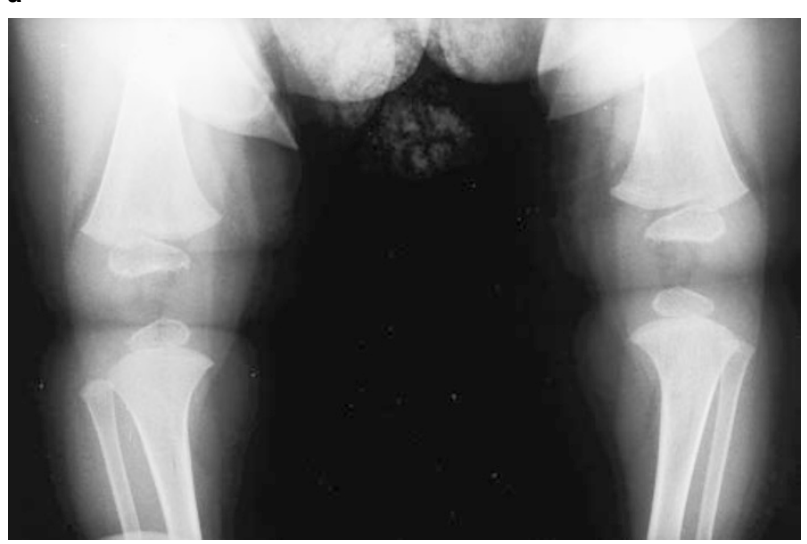

c

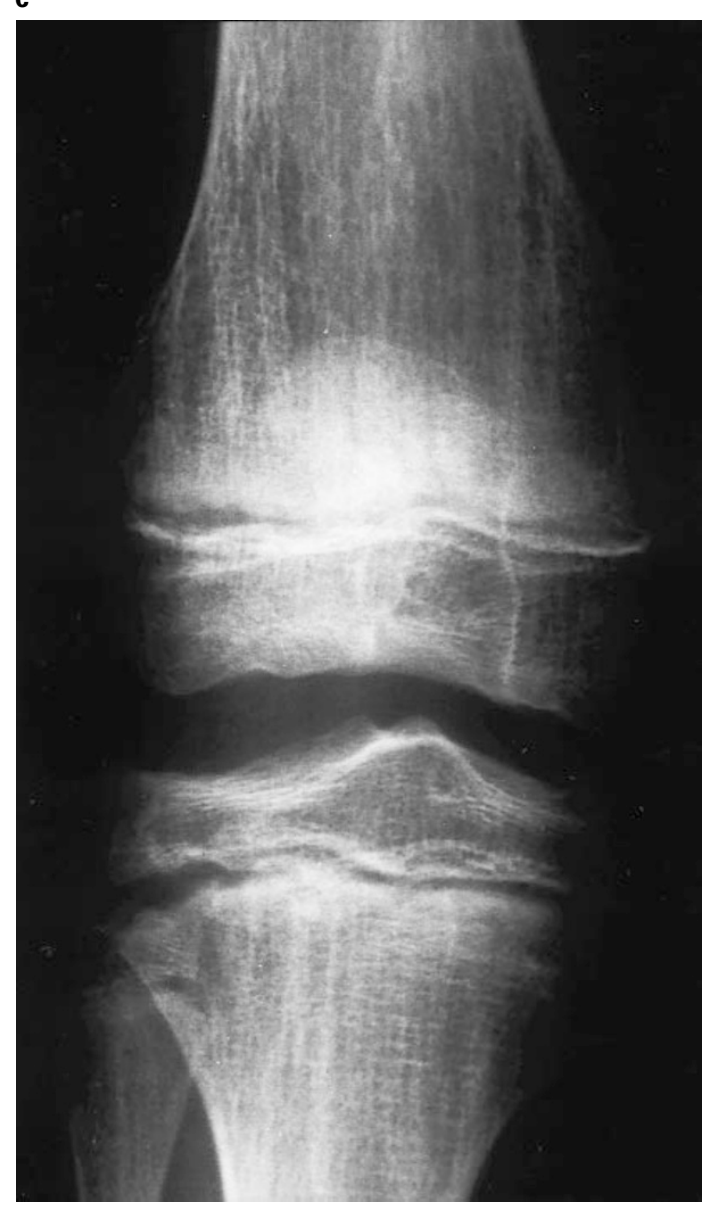

b

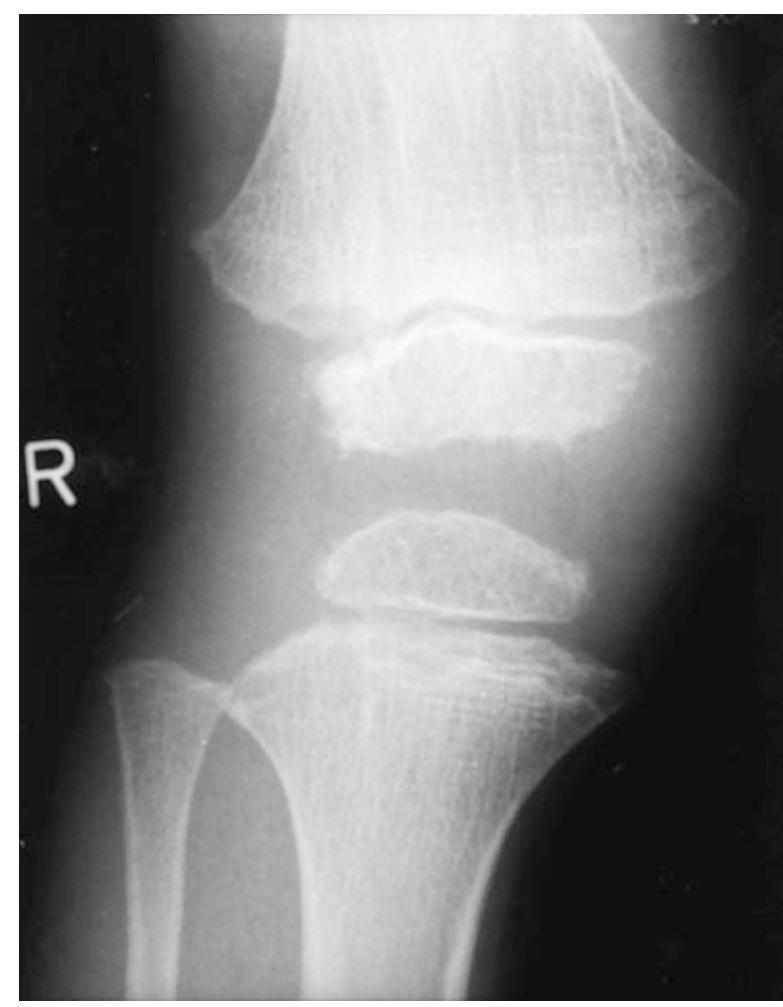

d

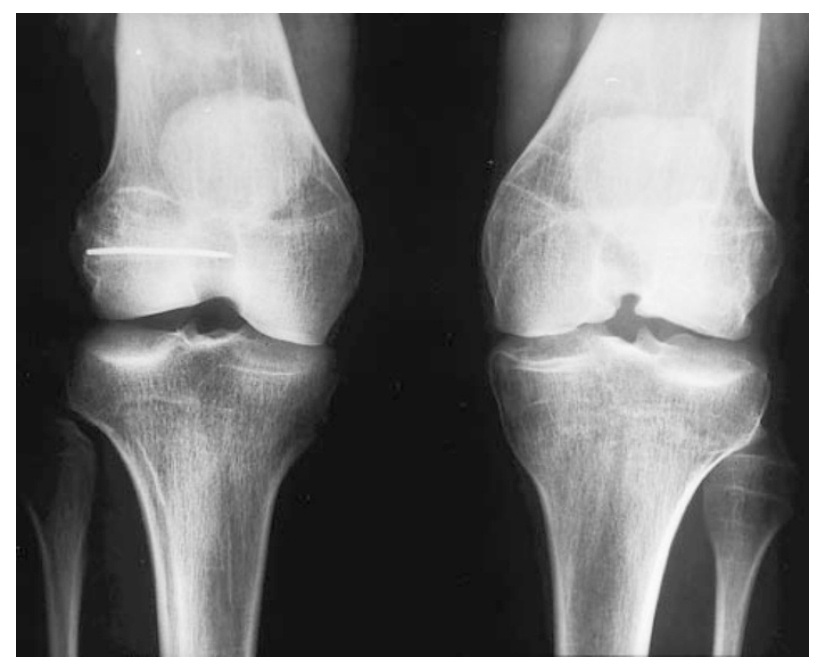

Figure 2 Radiographs of the knees of affected individuals at different ages. (a) AP film of the knees of individual IV-2 at 8 months of age. Note small epiphyses with irregular contours. (b) AP view of the right knee of IV-1 at 5 years of age shows absent ossification of the fibular epiphysis, small tibial and femoral epiphyses with irregular contours (most pronounced on the distal femoral epiphysis), abnormal tibial metaphysis with lateral ossification defect and irregular, translucent border. The femoral metaphysis is also irregular with longitudinal striations. (c) AP film of the right knee of III-2 at 14 years of age demonstrates flattened epiphyses and longitudinal metaphyseal striations, most pronounced in the distal femoral metaphysis. (d) Dysplasia and arthrosis of the knees in individual II-8 at 29 years of age. 
closely linked polymorphic marker for COL9A3. The only markers available were a single nucleotide polymorphism (SNP) with low heterozygosity and anonymous markers (D20S171 and D20S93), not closely linked to COL9A3. To identify a suitable polymorphic marker close to $C O L 9 A 3$, we analysed the sequence of a $170148 \mathrm{bp}$ genomic BAC clone (Locus HS885L7/Accession AL035669), which itself contained the entire COL9A3 gene. Sequence analysis identified numerous microsatellite repeats, one of which $\left[(\mathrm{CA})_{24}\right]$ was located approximately $15 \mathrm{~kb}$ to the $5^{\prime}$ end of exon 1 of COL9A3. Oligonucleotide primers, flanking the CA repeat, were designed to amplify a $196-214 \mathrm{bp}$ polymorphic fragment. A similar analysis was done on a clone containing the entire COL9A2 gene (Locus HSDJ39G22/Accession AL050341) and resulted in the identification of an appropriate microsatellite repeat ((atta/gt $\left.)_{15}\right)$, located approximately $29 \mathrm{~kb}$ to the $5^{\prime}$ end of exon 1 of COL9A2 (Table 2).

Genotypes were generated at each polymorphic marker and based on discordant segregation of alleles amongst affected individuals, a mutation in any of the tested genes could be excluded as causing MED in this family (Figure 4). Two-point LOD scores calculated between the disease phenotype and each of the markers confirmed that they were excluded, with a LOD score in excess of -2 at $\theta=0.001$ (Table 3). To generate two-point LOD scores, only those individuals who had undergone a comprehensive clinical and radiographic examination were included in the linkage calculation (II-2, II-7, II-8, III-2, III-3 and IV-1). Individuals II6 and II-9 were used to infer paternal genotypes only and their affection status was defined as unknown during linkage calculations. a

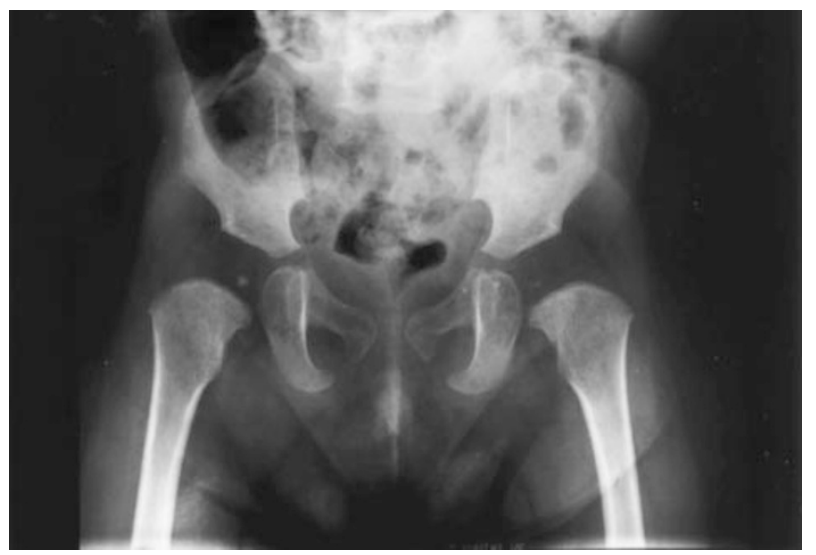

c

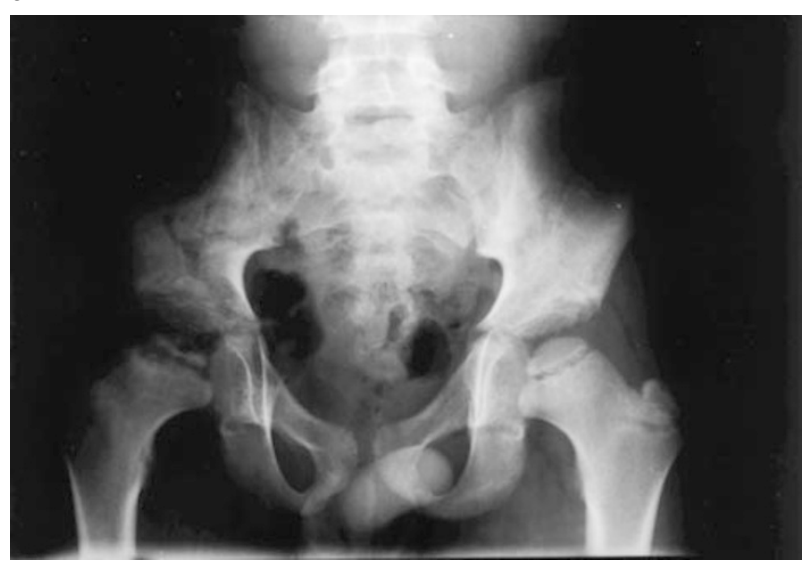

b

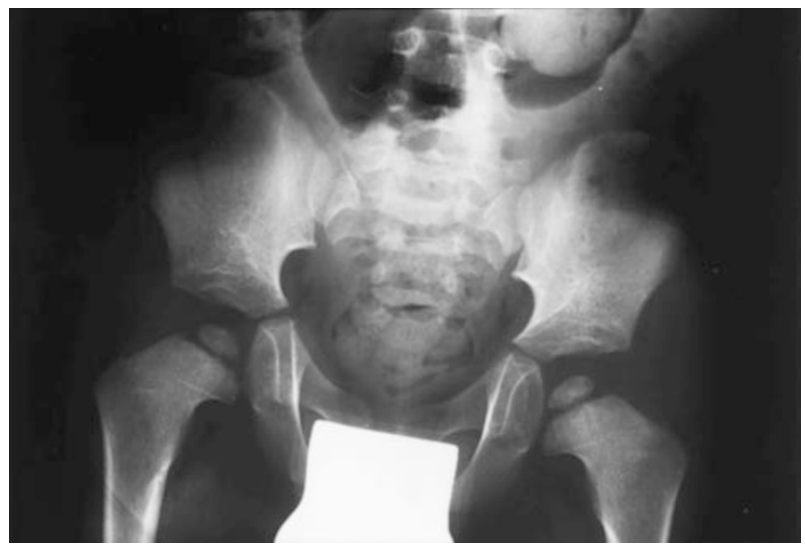

d

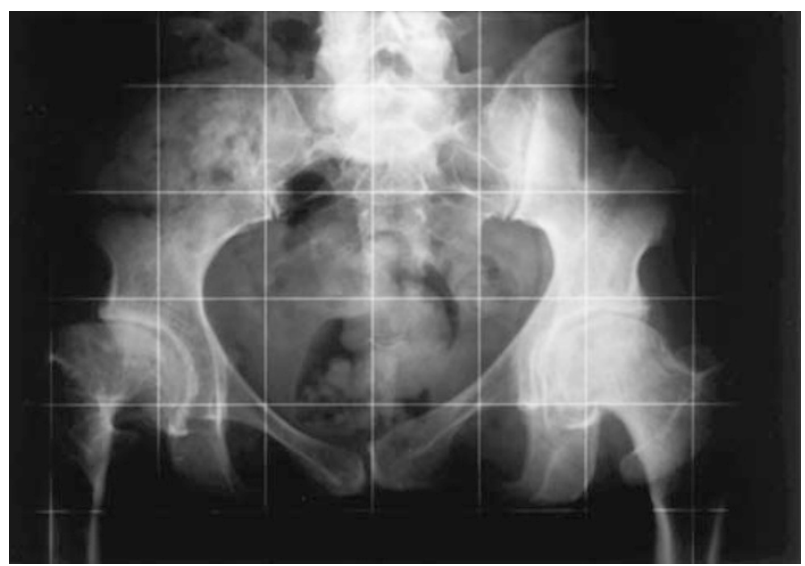

Figure 3 Radiographs of the pelvis of affected individuals at different ages. (a) Anteroposterior (AP) view of the pelvis of individual IV-2 at 19 months of age shows very small proximal femoral epiphyses. (b) AP film of individual IV- 1 at 5 years of age. The pelvis is characterised by flat acetabular roofs, small femoral epiphyses and absent ossification of the greater trochanters. (c) The pelvis of individual III-3 at 12 years of age shows small proximal femoral epiphyses with fragmentation on the right side and irregular acetabuli. (d) AP view of the pelvis in individual II-7 at the age of 46 years reveals coxarthrosis with severe deformation of the right femoral head and neck. 


\section{I}

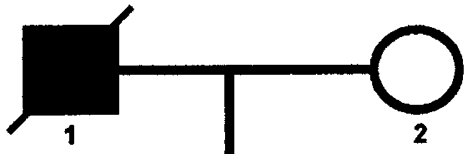

$\begin{array}{llll}\text { COL9A1 } & 12 B 1 & (171 & 169 \\ \text { COL9A1 } & 8 B 2 & (184 & 184 \\ \text { COL9A1 } & \text { (ttta) } & (290 & 290 \\ \text { COL9A2 } & \text { L-myc } & (190 & 195 \\ \text { COL9A2 } & \text { (atta)gt) } & (238 & 232 \\ \text { COL9A3 } & \text { (CA) } & (204 & 200 \\ \text { COMP } & \text { (taaa) } & (216 & 241 \\ \text { COL2A1 } & \text { (VNTR) } & \text { (A3 } & \text { A4 }\end{array}$

II

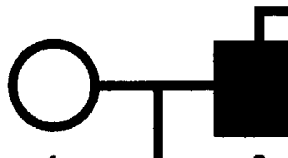

1

2

163171

184186

286290

167182

234238

206204

239249

A1 $\mathbf{A 1}$

\section{1}

184184

286290

178190

244238

204204

216235

A2 $\mathbf{A 3}$

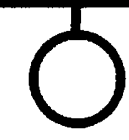

6

171171

184184

290286

184195

244232

20020

235241

A1 $\mathrm{A4}$
$167 \quad 171$

182184

290286

184178

244228

204204

235257

A1 A2

III

IV
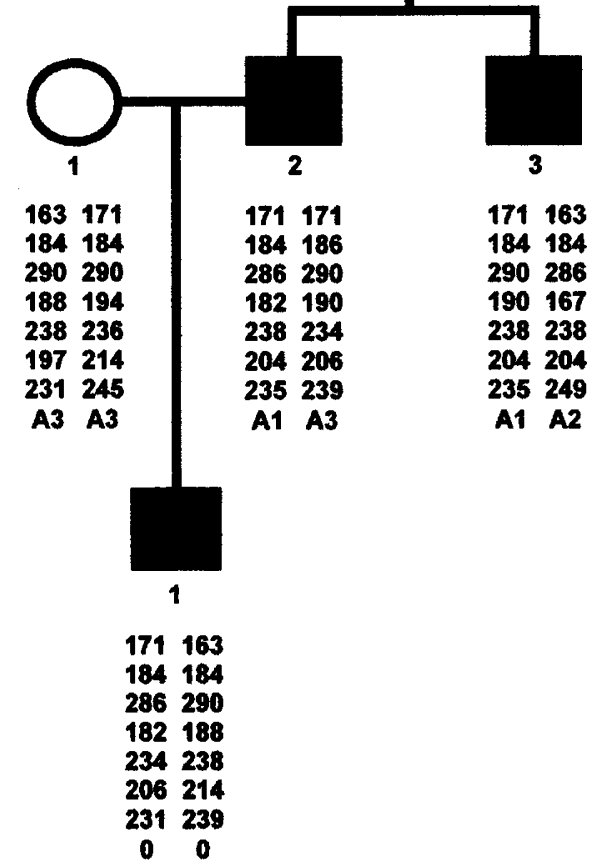

Figure 4 Pedigree of MED family showing genotypes generated at markers for each candidate gene. 
Table 1 Microsatellite markers associated with candidate genes for MED

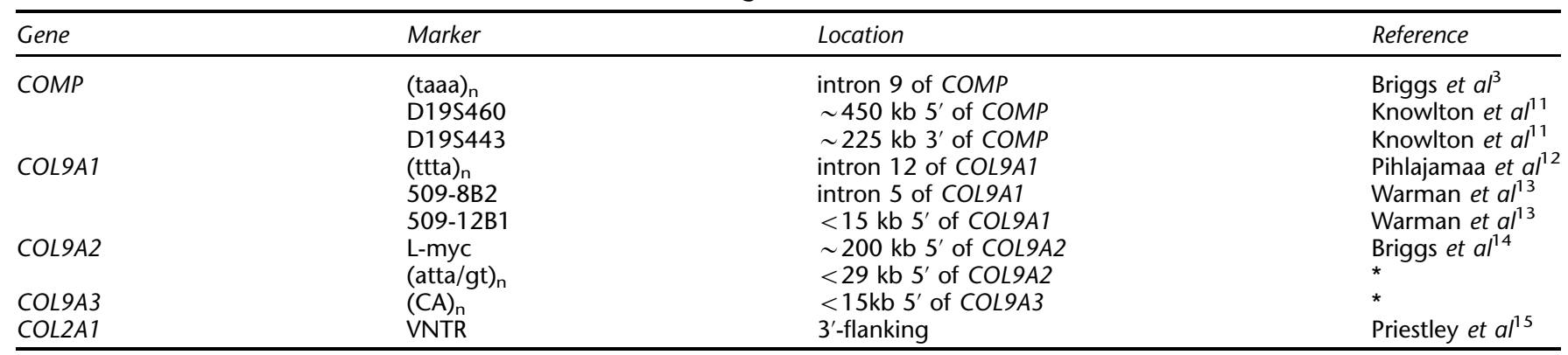

*Markers identified during this study (see also Table 2)

Table 2 Size of the PCR product and frequency of the alleles (calculated using 62 chromosomes) of the newly identified markers

\begin{tabular}{|c|c|c|c|c|c|}
\hline \multicolumn{3}{|c|}{ COL9A3 } & \multicolumn{3}{|c|}{ COL9A2 } \\
\hline Allele & Size $(b p)$ & Frequency & Allele & Size $(b p)$ & Frequency \\
\hline 1 & 193 & 0.016 & 1 & 230 & 0.048 \\
\hline 2 & 195 & 0.016 & 2 & 232 & 0.032 \\
\hline 3 & 197 & 0.016 & 3 & 234 & 0.097 \\
\hline 4 & 199 & 0.209 & 4 & 237 & 0.193 \\
\hline 5 & 201 & 0.1774 & 5 & 240 & 0.226 \\
\hline 6 & 203 & 0.1774 & 6 & 242 & 0.113 \\
\hline 7 & 205 & 0.161 & 7 & 246 & 0.065 \\
\hline 8 & 207 & 0.097 & 8 & 248 & 0.065 \\
\hline 9 & 209 & 0.065 & 9 & 250 & 0.065 \\
\hline 10 & 211 & 0.048 & 10 & 252 & 0.065 \\
\hline 11 & 213 & 0.016 & 11 & 258 & 0.016 \\
\hline
\end{tabular}

\section{Discussion}

We report a three-generation family with several relatives affected by multiple epiphyseal dysplasia. The disorder shows autosomal dominant inheritance. The condition is characterised clinically by complaints of easy fatigue and joint pain, mainly in the knees and hips, starting in early childhood. Stature is normal but signs of osteoarthrosis present early in adulthood. One patient had a hip replacement at the age of 47 years, another underwent knee replacement at 58 years. Radiographic abnormalities are already observed at the age of 8 months, before the onset of clinical symptoms. The epiphyses are primarily involved but the metaphyses also appear mildly abnormal. Before puberty, the epiphyses in the large joints are small and irregular, with both hips and knees mainly affected. In early childhood, the hand radiographs show small carpal bones with normal contours. Significant brachydactyly is absent and double layered patellae not observed. Except for Schmörl noduli in some of the affected individuals, the vertebral column remains normal. We compared the radiographic features of the affected family members with those reported in other MED patients with a COMP, COL9A2 and COL9A3 mutation. ${ }^{17}$ The hip abnormalities in our family were less severe than those observed in patients with a COMP mutation. On the other hand, the hips were more affected than in patients with a type IX collagen defect. The knee abnormalities in our family did resemble more the COL9A2/COL9A3 phenotype, however, without progression with age. There was rather a tendency of improvement of the knee changes. Hand radiographs were quite normal. Although carpal bones were small for age, carpal irregularity (as seen in both COMP and COL9A2/ COL9A3 patients) was not observed. Significant brachymetacarpalia with small epiphyses was not present in our family.

Through genetic linkage studies we have shown that the MED phenotype in this family is not the result of a mutation in any one of the three genes (COMP, COL9A2 and COL9A3) known to cause the autosomal dominant forms of MED. ${ }^{3-7}$ The possibility of a mutation in COL9A1, a candidate gene for MED since it encodes the $\alpha 1$ (IX) chain of type IX collagen, was also excluded. Our data provide strong evidence that at least a fourth locus, distinct from COL9A1, is involved in autosomal dominant MED. This is consistent with the observation that in $\sim 50 \%$ of MED patients no mutation can be identified in the genes encoding the COMP monomer or one of the constituent $\alpha$-chains of type IX collagen ${ }^{9}$ (KL Chapman and MD Briggs (unpublished observations) and $\mathrm{L}$ Ala-Kokko (personal communication)). Approximately 30\% of all MED cases result from mutations in the COMP gene ${ }^{9}$ and although COMP appears to be the major MED locus so far identified, it still accounts for less than half of the MED cases.

This study is the first step in the identification process of another important gene causing MED. The characterisation of this gene will ultimately help us in elucidating the cause of MED in those cases in which the genetic defect still remains to be determined. Because of the dominant inheritance pattern of the phenotype, a gene coding for another structural component of the cartilage extracellular matrix, would be a good candidate. Moreover, a gene encoding a protein that interacts with the other proteins involved in MED, is not unlikely. COMP and type IX collagen have been shown to interact with high affinity and mutations in the type IX collagen genes have been reported to cause a reduction of COMP-type IX collagen interactions. ${ }^{18}$ In addition, the DTDST gene, causing the recessive form of 
Table 3 Two-point LOD scores calculated between the MED phenotype and genotypes generated at five candidate genes

\begin{tabular}{|c|c|c|c|c|c|c|c|c|}
\hline \multirow{2}{*}{ Gene } & \multicolumn{8}{|c|}{ Recombination fraction $(\theta)$} \\
\hline & 0.00 & 0.001 & 0.01 & 0.05 & 0.10 & 0.20 & 0.30 & 0.40 \\
\hline \multicolumn{9}{|l|}{ COL9A1 } \\
\hline $12 \mathrm{~B} 1$ & -5.33 & -3.31 & -2.22 & -1.27 & -0.80 & -0.36 & -0.14 & -0.03 \\
\hline $\begin{array}{r}(t t t a)_{n} \\
\text { COL9A2 }\end{array}$ & -5.63 & -2.22 & -1.23 & -0.57 & -0.32 & -0.12 & -0.04 & -0.01 \\
\hline L-myc & $-\infty$ & -4.22 & -2.24 & -0.93 & -0.44 & -0.06 & 0.05 & 0.06 \\
\hline$(\mathrm{atta} / \mathrm{gt})_{\mathrm{n}}$ & $-\infty$ & -4.50 & -2.51 & -1.16 & -0.63 & -0.18 & -0.01 & 0.04 \\
\hline COL9A3 & & & & & & & & \\
\hline$(\mathrm{CA})_{\mathrm{n}}$ & $-\infty$ & -4.82 & -2.83 & -1.49 & -0.94 & -0.47 & -0.24 & -0.10 \\
\hline $\begin{array}{l}(\text { taaa })_{\mathrm{n}} \\
\text { COL2A1 }\end{array}$ & $-\infty$ & -10.22 & -6.23 & -3.49 & -2.35 & -1.27 & -0.68 & -0.29 \\
\hline (VNTR) & $-\infty$ & -5.71 & -3.62 & -1.99 & -1.25 & -0.55 & -0.22 & -0.05 \\
\hline
\end{tabular}

MED, may be important in the sulphation of these different proteins. This all would explain locus heterogeneity in one particular skeletal dysplasia showing clinical variability. Ultimately, the identification of all the genes causing MED may provide us with important insights into the protein interactions in cartilage extracellular matrix and open up new perspectives in the treatment of osteoarthrosis in the general population.

\section{Acknowledgments}

We thank the family for their very fruitful cooperation. The Flanders Fund for Scientific Research (Grant G.0013.97 to GR Mortier) and the Arthritis Research Campaign (Grant B0644 to MD Briggs) funded this work. MD Briggs is an ARC Research Fellow. Genotyping was performed in the ARC Epidemiology Unit, within the School of Epidemiology and Health Science, University of Manchester.

\section{References}

1 Rimoin DL, Lachman RS: Genetic disorders of the osseous skeleton; in Beighton P (ed): Heritable Disorders of Connective Tissue. St. Louis, 1993, pp 557-689.

2 Taybi H, Lachman RS: Radiology of Syndromes, Metabolic Disorders, and Skeletal Dysplasias. St. Louis, Mosby, 1996.

3 Briggs MD, Hoffman SMG, King LM et al: Pseudoachondroplasia and multiple epiphyseal dysplasia due to mutations in the cartilage oligomeric matrix protein gene. Nature Genet 1995; 10: $330-336$.

4 Briggs MD, Mortier GR, Cole WG et al: Diverse mutations in the gene for cartilage oligomeric matrix protein (COMP) in the pseudoachondroplasia-multiple epiphyseal dysplasia disease spectrum. Am J Hum Genet 1998; 62: 311-319.

5 Hecht JT, Nelson LD, Crowder E et al: Mutations in exon 17B of cartilage oligomeric matrix protein (COMP) cause pseudoachondroplasia. Nature Genet 1995; 10: 325-329.

6 Holden P, Canty EG, Mortier GR et al: Identification of novel pro- $\alpha 2$ (IX) collagen gene mutations in two families with distinctive oligo-epiphyseal forms of multiple epiphyseal dysplasia. Am J Hum Genet 1999; 65: 31-38.
7 Paassilta P, Lohiniva J, Annunen S et al: COL9A3: a third locus for multiple epiphyseal dysplasia. Am J Hum Genet 1999; 64: $1036-1044$.

8 Superti-Furga A, Neumann L, Riebel T et al: Recessively inherited multiple epiphyseal dysplasia with normal stature, club foot, and double layered patella caused by a DTDST mutation. $J$ Med Genet 1999; 36: 621-624.

9 Unger SL, King LM, Sobetzko D et al: A multiplicity of loci for multiple epiphyseal dysplasia. Am J Hum Genet 2000; 67 (Suppl 2): 371 .

10 Deere M, Blanton SH, Scott CI, Langer LO, Pauli RM, Hecht JT: Genetic heterogeneity in multiple epiphyseal dysplasia. Am J Hum Genet 1995; 56: 698-704.

11 Knowlton RG, Cekleniak JA, Cohn DH et al: High-resolution genetic and physical mapping of multiple epiphyseal dysplasia and pseudoachondroplasia mutations at chromosome 19p13.1p12. Genomics 1995; 28: $513-519$.

12 Pihlajamaa T, Vuoristo MM, Annunen S et al: Human COL9A1 and COL9A2 genes. Two genes of 90 and $15 \mathrm{~kb}$ code for similar polypeptides of the same collagen molecule. Matrix Biol 1998; 17: $237-241$.

13 Warman ML, Tiller GE, Polumbo PA et al: Physical and linkage mapping of the human and murine genes for the alpha 1 chain of type IX collagen (COL9A1). Genomics 1993; 17: 694-698.

14 Briggs MD, Choi H, Warman ML et al: Genetic mapping of a locus for multiple epiphyseal dysplasia (EDM2) to a region of chromosome 1 containing a type IX collagen gene. Am J Hum Genet 1994; 55: 678-684.

15 Priestley L, Kumar D, Sykes B: Amplification of the COL2A1 3 variable region used for segregation analysis in a family with the Stickler syndrome. Hum Genet 1990; 85: 525-526.

16 Kuivaniemi H, Tromp G, Prockop DJ: Mutations in fibrillar collagens (types I, II, III, and XI), fibril-associated collagen (type IX), and network-forming collagen (type X) cause a spectrum of disease of bone, cartilage, and blood vessels. Hum Mutat 1997; 9 : $300-315$.

17 Unger SL, Briggs MD, Holden P et al: Multiple epiphyseal dysplasia: radiographic abnormalities correlated with genotype. Pediatr Radiol 2001; 31: 10-18.

18 Holden P, Meadows RS, Chapman KL et al: Cartilage oligomeric matrix protein interacts with type IX collagen; disruptions to these interactions identify a pathogenetic mechanism in a bone dysplasia family. J Biol Chem 2001; 216: 6046-6055. 